\title{
Comparison and Validation of Compressible Flow Simulations of Laser-Induced Cavitation Bubbles *
}

\author{
Siegfried Müller ${ }^{\mathrm{a}}$ Mathieu Bachmann ${ }^{\mathrm{a}}$ Dennis Kröninger ${ }^{\mathrm{b}}$ \\ Thomas Kurz ${ }^{\mathrm{b}}$ Philippe Helluy ${ }^{\mathrm{c}}$ \\ ${ }^{a}$ Institut für Geometrie und Praktische Mathematik, RWTH Aachen, 52056 \\ Aachen, Germany \\ ${ }^{\mathrm{b}}$ Drittes Physikalisches Institut, Universität Göttingen, Germany \\ ${ }^{\mathrm{c}}$ Institut de Recherche Mathématique Avancée, Université de Louis Pasteur, \\ Strasbourg, France
}

\begin{abstract}
The numerical simulation of compressible two-phase fluid flows exhibits severe difficulties, in particular, when strong variations in the material parameters and high interface velocities are present at the phase boundary. Although several models and discretizations have been developed in the past, a thorough quantitative validation by experimental data and a detailed comparison of numerical schemes are hardly available.

Here two different discretizations are investigated, namely, a non-conservative approach proposed by Saurel and Abgrall (SIAM J. Sci. Comput. 21, 1115 (1999)) and the real ghost fluid method developed by Tang, Liu and Khoo (SIAM J. Sci. Comput. 28, 278 (2006)). The validation is performed for the case of laser-induced cavitation bubbles collapsing in an infinite medium. For the computations, initial data are deduced implicitly from the experimental data. In particular, the influence of numerical phase transition caused by smearing of the phase boundary is investigated.
\end{abstract}

Key words: spherical bubble collapse, stiffened gas model, ghost fluid method

ऋ This work has been performed with funding by the Deutsche Forschungsgemeinschaft in the DFG-CNRS-Forschergruppe FOR 563 "Micro-Macro Modeling and Simulation of Liquid-Vapor Flows"

Email address: mueller@igpm.rwth-aachen.de (Siegfried Müller).

URL: http://www.igpm.rwth-aachen.de/mueller (Siegfried Müller). 


\section{Introduction}

The investigation of cavitation bubbles is of interest for different real world applications arising, for instance, in engineering, medicine and biology. The processes taking place in the interior and exterior of a collapsing and oscillating bubble are still subject of theoretical and experimental research [2]. However, small time and space scales as well as the complicated dynamics make any theoretical and experimental approach a challenge.

In the present work we will focus on the modeling and simulation of the collapse and rebound of a single vapor-filled spherical bubble in a liquid environment. This configuration has been subject of numerous analytical and experimental investigations. An overview of the field is given in the review article by Lauterborn el al. [14].

Numerical investigations have verified that the fluid state inside the bubble does not stay homogeneous during the collapse [8]. Moreover, shock waves develop in the liquid when the bubble gets maximally compressed, as is confirmed by experiments [2]. Presumably, strong waves are also generated inside the bubble, where they may interact with the phase boundary due to the small radius. Thereby, the frequently made assumption of incompressibility of the liquid and the homogeneity of the bubble medium are inappropriate when considering strong bubble collapse. In addition it could be verified that the modeling of the gas inside the bubble by a perfect gas is only valid at moderate changes in volume [8].

In recent years, several numerical investigations on compressible two-phase fluid flow for even more complex configurations have been published using the stiffened gas law $[4-6,15,16,25]$. This model was introduced by Harlow and Amsden [9] and can be considered a combination of the perfect gas law and the barotrop Tait equation supplemented with an appropriate energy law [22]. Here the material parameters depend on the phase.

For the numerical discretization of the Euler equations equipped with the stiffened gas law, different approaches have been used, for instance, the real ghost fluid method [25], a characteristics-based matching scheme [16] and a stratified flow model [6]. The main objective of this work is to compare two different discretizations, namely, (i) the Saurel and Abgrall (SA) approach [19] and (ii) the real ghost fluid method (rGFM) [25]. Both rely on a finite volume discretization of the Euler equations that only differs in the flux computation at cell interfaces next to the phase boundary. Here the SA approach uses a two-phase Riemann solver whereas in the rGFM two single-phase Riemann problems with appropriate values for the ghost fluid are solved. The main difference inherent in the discretizations is the choice of the phase indicator 
function, namely, (i) the gas fraction in the SA approach and (ii) the level set function in the rGFM. The evolution of both phase indicator functions is described by the same non-conservative evolution equation but its discretization differs significantly: The evolution equation for the gas fraction is discretized by a non-conservative upwind scheme adapted to the underlying finite volume discretization of the flow equations. On the other hand, for the discretization of the level set function well-known techniques are applied to the transport equation. To ensure the property of a distance function the level set function is reinitialized in a postprocessing step.

The two discretizations are to be compared by means of quasi-1D computations of a spherical bubble in order to investigate the influence of numerical phase transition. We do this by means of the physically relevant configuration of laser-induced cavitation bubbles. One problem is the choice of initial data that can not be directly deduced from the experiment. Therefore another focus of the present work is to suggest a strategy how to determine initial data that are implicitly fitted to the time evolution of the measured bubble radius.

The outline is as follows. In Section 2 we summarize the stiffened gas model. This model is discretized by the SA approach as well as the rGFM summarized in Section 3. Then, in Section 4, we propose a strategy for the computation of the initial data fitted to experimental data that are provided by the collapse of a laser-induced spherical bubble. Finally, in Section 5, we present several numerical computations for two test cases corresponding to a small and a large equilibrium radius of the bubble. In particular, we compare the two different discretizations and validate them by the experimental data.

\section{Mathematical Model}

Compressible fluid flow is characterized in continuum mechanics by the fields of density $\rho$, velocity $\mathbf{v}$, internal energy $e$ and pressure $p$. The balances of mass, momentum and energy for inviscid flow lead to the Euler equations. In spherical coordinates and employing rotational symmetry, they read

$$
\begin{aligned}
& \frac{\partial}{\partial t}\left(r^{2} \rho\right)+\frac{\partial}{\partial r}\left(r^{2}\left(\rho v_{r}\right)\right)=0 \\
& \frac{\partial}{\partial t}\left(r^{2} \rho v_{r}\right)+\frac{\partial}{\partial r}\left(r^{2}\left(\rho v_{r}^{2}+p\right)\right)=2 p r \\
& \frac{\partial}{\partial t}\left(r^{2} \rho E\right)+\frac{\partial}{\partial r}\left(r^{2}\left(\rho v_{r}(E+p / \rho)\right)\right)=0
\end{aligned}
$$

where $E=e+\frac{1}{2} v_{r}^{2}$ is the total energy and $v_{r}$ the radial velocity. 
In order to close the system, we have to provide a pressure law. In this work, we consider the stiffened gas law suggested in $[1,7]$. It reads

$$
p(\rho, e, \varphi)=(\gamma(\varphi)-1) \rho e-\gamma(\varphi) \pi(\varphi)
$$

where the two phases (here a gas and a liquid) are distinguished by an additional unknown $\varphi$. Note that with $\varphi=0$ we recover a standard perfect gas law. In the following we will consider two choices for the phase indicator function $\varphi$. It will represent either the gas fraction or the level set function.

\subsection{Evolution of phase boundary by gas fraction}

In this approach we identify $\varphi$ with the gas fraction. We decide that $\varphi=0$ and $\varphi=1$ correspond to pure liquid and vapor, respectively. Because we are interested in very high speed flows and very short observation times we suppose that phase transition can be neglected so that there is no mass transfer between the two fluids. Thus the fraction satisfies a homogeneous transport equation, i.e., its material derivative is vanishing,

$$
\frac{\partial \varphi}{\partial t}+v_{r} \frac{\partial \varphi}{\partial r}=0
$$

If at initial time $t=0$ the fraction $\varphi$ takes only the values 0 or 1 , it will retain its value for $t>0$. Thus there is no physical mixing in the continuous model. However, the numerical model will introduce artificial mixture zones where $0<\varphi<1$. This causes some difficulties that are discussed later in this section.

If we were only studying the continuous model, it would be sufficient to provide the values of the pressure law coefficients $\gamma$ and $\pi$ for $\varphi=0$ or $\varphi=1$. But because of the numerical mixture, it is necessary to interpolate $\gamma$ and $\pi$ for $0<\varphi<1$. An arbitrary choice of interpolation would lead to numerical difficulties that are studied in many works, see for instance Ref. [1]. It appears that a good choice consists in a linear interpolation of the two special quantities $\beta_{1}=1 /(\gamma-1)$ and $\beta_{2}=\gamma \pi /(\gamma-1)$, i.e.,

$$
\beta_{1}(\varphi)=\varphi \beta_{1}(1)+(1-\varphi) \beta_{1}(0), \quad \beta_{2}(\varphi)=\varphi \beta_{2}(1)+(1-\varphi) \beta_{2}(0) .
$$

The mixture pressure law coefficients $\gamma(\varphi)$ and $\pi(\varphi)$ are then obtained from the reverse relation, i.e.,

$$
\gamma(\varphi)=1+1 / \beta_{1}(\varphi), \quad \pi=\beta_{2}(\varphi) /\left(1+\beta_{1}(\varphi)\right)
$$


Table 1

Material coefficients

\begin{tabular}{|l|l|l|l|}
\hline & & Vapor & Liquid \\
\hline$\gamma$ & {$[-]$} & 1.4 & 1.1 \\
$\pi$ & {$[\mathrm{Pa}]$} & 0 & $2.045 \mathrm{e}+9$ \\
\hline
\end{tabular}

For pure water and air the material parameters $\gamma$ and $\pi$ are listed in Table 1 . Here the minimal pressure $p_{\min }=-\pi$ for the pure liquid is deduced from the sound speed $c^{2}=\gamma(p+\pi) / \rho$ where we choose $c_{l}=1500 \mathrm{~m} / \mathrm{s}, \gamma_{l}=1.1$, $\rho_{l}=1000 \mathrm{~kg} / \mathrm{m}^{3}$ and $p_{l}=10^{5} \mathrm{~Pa}$. Usually in the stiffened gas model for a liquid, higher values of $\gamma_{l}$ are proposed as in Ref. [7]. However, smaller values for $\gamma_{l}$ result in a higher minimal pressure $\pi_{l}$ and, hence, the variation of the sound speed in the liquid is smaller.

\subsection{Evolution of phase boundary by level set function}

An alternative approach to distinguish between the two phases is based on the level set technique first proposed by Osher and Sethian $[17,20]$. The basic idea is to embed the phase boundary in a higher-dimensional manifold. In the quasi-1D case considered here it is characterized by the bubble radius $r_{I}$, i.e., $\Phi=\Phi(t, r)$, where at time $t$ the phase boundary is characterized by the zero level $\Phi\left(t, r_{I}\right)=0$.

The interface is evolved in time according to the fluid velocity $v_{r}$. Therefore, an observer sitting on the interface $r_{I}$ detects no change in $\Phi$ and, hence, a natural choice for the evolution of the level set function $\Phi$ is

$$
\frac{\partial \Phi}{\partial t}+v_{r} \frac{\partial \Phi}{\partial r}=0
$$

In order to identify the phase present at an arbitrary position $r$ by the sign of $\Phi$, we initialize $\Phi$ as the signed distance function to the interface, i.e.,

$$
\Phi(0, r)=\operatorname{sign}\left(r-r_{I}\right)\left|r-r_{I}\right|
$$

Note that $\Phi$ remains no longer a distance function with increasing time. In Ref. [23], Sussman et al. proposed a reinitialization method for the scalar field that preserves its character of a distance function. For this purpose they suggest to solve

$$
\frac{\partial \tilde{\Phi}}{\partial \tau}=S(\tilde{\Phi})\left(1-\left|\frac{\partial \Phi}{\partial r}\right|\right) \quad \operatorname{resp} . \quad \frac{\partial \tilde{\Phi}}{\partial \tau}+a(\tilde{\Phi}) \frac{\partial \tilde{\Phi}}{\partial r}=S(\tilde{\Phi})
$$


with $a=S(\tilde{\Phi}) \frac{\partial \tilde{\Phi}}{\partial r} /\left|\frac{\partial \tilde{\Phi}}{\partial r}\right|$ and $S=S(\tilde{\Phi})=\operatorname{sign}(\tilde{\Phi})$ the sign-function. Note that $\tau$ is an artificial time. Here we denote the reinitialization function by $\tilde{\Phi}$ to distinguish it from the level set function $\Phi$. It is initialized by

$$
\tilde{\Phi}(0, r)=\Phi(t, r)
$$

for some fixed time $t$. Thus, after each evolution step of the level set function the stationary solution to equation (8) is determined to ensure that $\Phi$ remains a distance function and that the zero level is kept. Note that the definition of the sign-function $S$ together with (8) guarantees that the zero level is preserved, i.e., $\tilde{\Phi}\left(\tau, r_{I}\right)=\Phi\left(t, r_{I}\right)=0$ for $\tau \geq 0$.

\section{Discretization}

In order to discretize the inviscid flow equations (1) and the evolution equations of the phase indicator function (3) and (6), respectively, we will employ the Saurel-Abgrall approach [19] and the real ghost fluid method [25]. These two approaches have in common that the flow equations for the conserved quantities are approximated by a second-order finite volume scheme in space and an upwind discretization is used for the non-conservative transport equations of the phase indicator. Due to the nonstationary behavior of the flow, time integration is performed explicitly. The efficiency of the resulting scheme is improved by applying multiscale-based grid adaptation techniques $[3,15]$.

The key ideas are briefly summarized below where, in particular, we address the main differences between the two approaches.

\subsection{The Saurel-Abgrall approach}

The main idea in the SA approach is to couple the discretization of the flow equations (1) with the gas fraction equation (3).

First of all, a finite volume discretization is applied to (1) that reads

$$
\boldsymbol{v}_{i}^{n+1}=\boldsymbol{v}_{i}^{n}-\frac{\Delta t}{\Delta r^{3}}\left(r_{i+\frac{1}{2}}^{2} \boldsymbol{F}_{i+\frac{1}{2}}^{n}-r_{i-\frac{1}{2}}^{2} \boldsymbol{F}_{i-\frac{1}{2}}^{n}\right)+\frac{\Delta r \Delta t}{\Delta r^{3}} \boldsymbol{S}_{i}^{n}
$$

for cell $I_{i}=\left[r_{i-\frac{1}{2}}, r_{i+\frac{1}{2}}\right]$ with $\Delta r:=r_{i+\frac{1}{2}}-r_{i-\frac{1}{2}}$ the spatial discretization length in radial direction. The volume is determined by $\Delta r^{3}:=\frac{1}{3}\left(r_{i+\frac{1}{2}}^{3}-r_{i-\frac{1}{2}}^{3}\right)$ and 
the interface area by $r_{i+\frac{1}{2}}^{2}$. Here $\boldsymbol{v}=\left(\rho, \rho v_{r}, \rho E\right)^{T}$ is an approximation of the cell averages of the conserved quantities and $\boldsymbol{F}_{i \pm \frac{1}{2}}^{n}$ denote the numerical fluxes at the interfaces $r_{i \pm \frac{1}{2}}$ approximating the flux in radial direction $\boldsymbol{F}_{r}=$ $\left(\rho v_{r}, \rho v_{r}^{2}+p, v_{r}(\varrho E+p)\right)^{T}$. The numerical flux is determined by (i) a secondorder reconstruction of the primitive variables $\rho, v_{r}, p$ and the gas fraction $\varphi$ and (ii) the exact solution of a Riemann problem. The numerical source term $\boldsymbol{S}_{i}^{n}$ is approximated by evaluating the right-hand side of Eq. (1) in the cell center $\hat{r}_{i}=\left(r_{i-\frac{1}{2}}+r_{i+\frac{1}{2}}\right) / 2$ with pressure $p_{i}^{n}$, i.e.,

$$
\boldsymbol{S}^{n}=\left(0,2 \hat{r}_{i} p_{i}^{n}, 0\right)
$$

This is consistent with $v_{r}=0$ and $p=$ const.

In order to avoid pressure and velocity oscillations at the phase boundary Saurel and Abgrall [19] suggested an upwind discretization of the evolution equation for the fraction $\varphi$. It is derived from the finite volume discretization for the homogeneous fluid equations, i.e., $\boldsymbol{S}_{i}^{n}=\mathbf{0}$ in Eq. (10), applied to a flow field exhibiting constant velocity and pressure states. In order to maintain these constant states the upwind discretization for $\varphi$ has to be chosen such that

$$
\varphi_{i}^{n+1}=\varphi_{i}^{n}-\frac{\Delta t}{\Delta r^{3}}\left(r_{i+\frac{1}{2}}^{2} \bar{v}_{r, i+\frac{1}{2}}^{n}\left(\bar{\varphi}_{i+\frac{1}{2}}^{n}-\varphi_{i}^{n}\right)-r_{i-\frac{1}{2}}^{2} \bar{v}_{r, i-\frac{1}{2}}^{n}\left(\bar{\varphi}_{i-\frac{1}{2}}^{n}-\varphi_{i}^{n}\right)\right)
$$

where $\bar{\varphi}$ and $\bar{v}_{r}$ are the gas fraction and the velocity given by the solution of the two-phase Riemann problem at the interface $i \pm \frac{1}{2}$. Alternatively, we could evolve the vector $\boldsymbol{\beta}=\left(\beta_{1}, \beta_{2}\right)^{T}$ of the material parameters by

$$
\boldsymbol{\beta}_{i}^{n+1}=\boldsymbol{\beta}_{i}^{n}-\frac{\Delta t}{\Delta r^{3}}\left(r_{i+\frac{1}{2}}^{2} \bar{v}_{r, i+\frac{1}{2}}^{n}\left(\overline{\boldsymbol{\beta}}_{i+\frac{1}{2}}^{n}-\boldsymbol{\beta}_{i}^{n}\right)-r_{i-\frac{1}{2}}^{2} \bar{v}_{r, i-\frac{1}{2}}^{n}\left(\overline{\boldsymbol{\beta}}_{i-\frac{1}{2}}^{n}-\boldsymbol{\beta}_{i}^{n}\right)\right) \cdot(13
$$

Note that the terms $\varphi_{i}^{n}$ and $\boldsymbol{\beta}_{i}^{n}$ in (12) and (13), respectively, would cancel for a homogeneous velocity field in the genuine multidimensional case but not for the quasi-1D case.

Finally we summarize the numerical discretization of the Saurel-Abgrall approach. It consists of the following actions at each time step:

(1) compute the reconstruction of the primitive variables,

(2) solve the Riemann problem for each cell interface,

(3) evolve density, momentum and energy in time by (10),

(4) evolve the gas fraction in time by (12) where the transport velocities at each cell interface are determined by the Riemann solutions. 


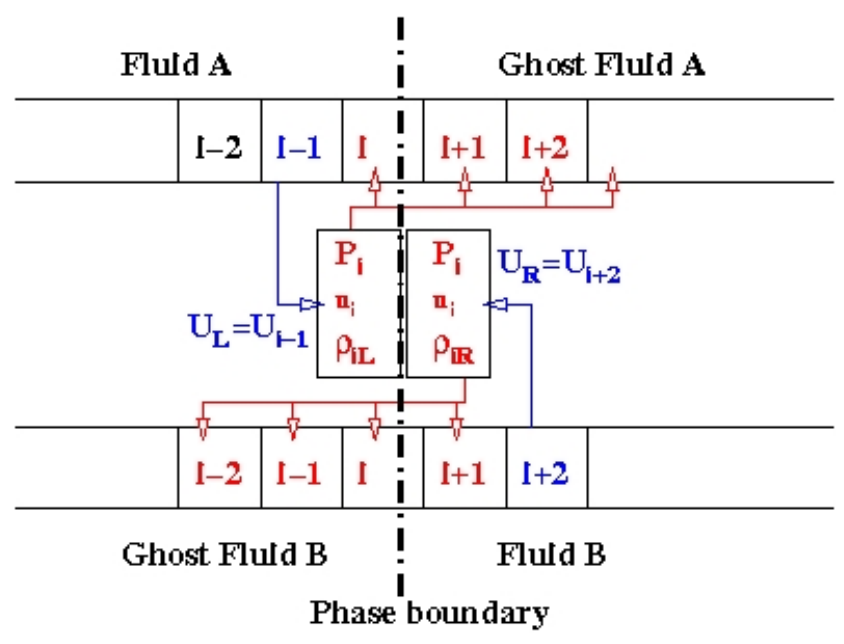

Fig. 1. Sketch of the computation of the real and ghost fluid states from the interfacial states $u_{I}, p_{I}$ and $\rho_{I L}, \rho_{I R}$ determined by solving a two-phase Riemann problem for the states $u_{L}$ and $u_{R}$.

\subsection{The real ghost fluid method}

In this approach the fluid equations are also discretized by a finite volume approximation

$$
\boldsymbol{v}_{i}^{n+1}=\boldsymbol{v}_{i}^{n}-\frac{\Delta t}{\Delta r_{i}^{3}}\left(r_{i+\frac{1}{2}}^{2} \boldsymbol{F}_{i+\frac{1}{2}}^{n,-}-r_{i-\frac{1}{2}}^{2} \boldsymbol{F}_{i-\frac{1}{2}}^{n,+}\right)+\frac{\Delta r_{i} \Delta t}{\Delta r_{i}^{3}} \boldsymbol{S}_{i}^{n}
$$

that is similar to (10) but near to the phase boundary we define two fluxes $\boldsymbol{F}_{i+\frac{1}{2}}^{n, \pm}$, i.e., the resulting scheme is not conservative. These fluxes are determined by first solving a two-phase Riemann problem. If the phase boundary is lying between cell $i$ and cell $i+1$ where cell $i$ corresponds to fluid $A$ and cell $i+1$ to fluid $\mathrm{B}$, then the left and right state are taken from cells $i-1$ and $i+2$, respectively, to ensure access to states of the pure phases. The Riemann solution provides interfacial states for density $\rho_{I L}, \rho_{I R}$, pressure $p_{I}$ and velocity $v_{I}$ left and right to the phase boundary. For fluid $\mathrm{A}$, the state of cell $i$ is substituted by $p_{I}, u_{I}, \rho_{I L}$ and also for the ghost cells on the other side of the phase boundary. Then a single-phase Riemann problem is solved for each cell interface of fluid A to provide the numerical fluxes with the ghost cells as boundary conditions at the phase boundary. Then the same procedure is used for fluid B. This procedure is sketched in Fig. 1. After having computed the numerical fluxes, the solution is advanced to the next time step by Eq. (14). Note that away from the phase boundary only one numerical flux if computed at a cell interface by solving a single-phase Riemann problem. Again the spatial order is improved by using a second-order reconstruction of the primitive variables $\rho, v_{r}, p$ as well as the level set function $\Phi$. 
Opposite to the SA approach the evolution equation (6) of the level set function is discretized independently of the finite volume discretization using standard techniques. For the sake of completeness they are briefly summarized in the following. Here we employ a first-order time discretization

$$
\Phi_{i}^{n+1}=\Phi_{i}^{n}-\Delta t v_{i}^{n}(\partial \Phi / \partial r)_{i}^{n}
$$

where the spatial derivative is approximated by the upwind discretization

$$
(\partial \Phi / \partial r)_{i}=\left\{\begin{array}{ll}
m(A, B) & , A \cdot B \geq 0 \\
0 & , \text { else }
\end{array} .\right.
$$

Here, the terms $A$ and $B$ correspond to a second-order approximation of $\partial \Phi / \partial r$ according to an ENO discretization, see Harten et al. [10]. They are determined by

$$
\begin{aligned}
& A=D^{-} \Phi_{i}+\Delta r_{i} / 2 \cdot m\left(D^{+} D^{-} \Phi_{i}, D^{+} D^{-} \Phi_{i-1}\right), \\
& B=D^{+} \Phi_{i}-\Delta r_{i} / 2 \cdot m\left(D^{+} D^{-} \Phi_{i}, D^{+} D^{-} \Phi_{i+1}\right),
\end{aligned}
$$

using the minmod function $m(a, b)=a$, if $|a|<|b|$ and $m(a, b)=b$ else. The one-sided approximations $D^{+}$and $D^{-}$and the central difference $D^{+} D^{-}$of the first and second derivative of $\Phi$ are defined by

$D^{+} \Phi_{i}=\frac{\Phi_{i+1}-\Phi_{i}}{\Delta r_{i}}, D^{-} \Phi_{i}=\frac{\Phi_{i}-\Phi_{i-1}}{\Delta r_{i}}, D^{+} D^{-} \Phi_{i}=\frac{\Phi_{i+1}-2 \Phi_{i}+\Phi_{i-1}}{\left(\Delta r_{i}\right)^{2}}$

Note that the upwind discretization has a stabilizing effect similar to finite volume discretizations of conservation laws. Due to the transport, the gradients may steepen and the level set function develops discontinuities. In that case, it is no longer a distance function.

In order to maintain smoothness of the level set function and to preserve its character of a distance function, it is reinitialized after each time step. For this purpose we approximate the steady state solution of the initial value problem (8) and (9). For the spatial discretization an upwind discretization as proposed by Sussman et al. [24] is applied whereas in time we use a first order approximation, i.e.,

$$
\tilde{\Phi}_{i}^{n+1}=\tilde{\Phi}_{i}^{n}-\Delta \tau S_{\epsilon}\left(\tilde{\Phi}_{i}^{n}\right)\left(1-\left|(\partial \tilde{\Phi} / \partial r)_{i}^{n}\right|\right) .
$$


Here $S_{\epsilon}(\tilde{\Phi})=2\left(H_{\epsilon}(\tilde{\Phi})-1 / 2\right)$ is the smoothed sign function defined by

$$
H_{\epsilon}(\tilde{\Phi}):=\left\{\begin{array}{cl}
0 & , \tilde{\Phi}<-\epsilon \\
\frac{1}{2}\left(1+\frac{\tilde{\Phi}}{\epsilon}+\frac{1}{\pi} \sin \left(\frac{\pi \tilde{\Phi}}{\epsilon}\right)\right), & |\tilde{\Phi}| \leq \epsilon \\
1 & , \tilde{\Phi}>\epsilon
\end{array} .\right.
$$

Note that the smoothed Heaviside function $H_{\epsilon}$ guarantees that the zero level set is reproduced. Herein, $\epsilon$ is chosen to be three times the discretization length on the finest resolution in the grid. The reinitialization step needs a pseudo time $\tau$ depending on the discretization, here we use $\Delta \tau=0.3 \Delta r$. Unlike in Eq. (16) the spatial derivative $\partial \tilde{\Phi} / \partial r$ is approximated - as proposed by Shu and Osher [21] - by an upwind ENO-type scheme, i.e.,

$$
(\partial \tilde{\Phi} / \partial r)_{i}=\left\{\begin{array}{l}
A, w_{l}>0 \text { and } w_{l}+w_{r}>0 \\
B, w_{r}<0 \text { and } w_{l}+w_{r}<0 \\
0, \text { else }
\end{array}\right.
$$

with $w_{l}=S_{\epsilon}(\tilde{\Phi}) \cdot A, w_{r}=S_{\epsilon}(\tilde{\Phi}) \cdot B$ and $A, B$ defined according to Eq. (17).

Finally we summarize the numerical discretization of the real ghost fluid method. It consists of the following actions at each time step:

(1) solve a two-phase Riemann problem to determine the interfacial states at the phase boundary,

(2) determine the states in the ghost cells and modify the real fluid by the interfacial states,

(3) compute the reconstruction of the primitive variables,

(4) solve the single-phase Riemann problems at the cell interfaces,

(5) evolve density, momentum and energy in time by (14)

(6) evolve the level set function in time by (15) where the transport velocities are determined by the new velocities,

(7) reinitialize the level set function by some iteration steps with (20).

\section{Computation of initial data}

In order to start the computation we need initial data not only for the liquid state but also for the medium inside the bubble. However, it is not yet possible to measure the pertaining variables inside the bubble. Therefore we propose an indirect way to obtain the initial pressure and temperature in the bubble from 
the experimentally measured radius vs. time curve. The solution of a secondorder ODE (the Keller-Miksis model) describing the radial bubble dynamics is fitted to the measured data points to find consistent initial conditions.

\subsection{Laser-induced cavitation bubbles}

Cavitation bubbles can be produced by laser pulses. The experiments described here have been performed with a Q-switched Nd:YAG laser (Lumonics HY750). This laser delivers pulses at a wavelength of $1064 \mathrm{~nm}$ having a duration of $8 \mathrm{~ns}$. After tuning the energy to a few mJ by an attenuator, the laser pulse is focused into a cuvette of size $50 \times 50 \times 50 \mathrm{~mm}^{3}$ filled with clean, distilled water. The liquid temperature is kept fairly constant at $20^{\circ}$ $\mathrm{C}$ during the experiment. A lens system built into the cuvette produces a breakdown plasma at a distance of about $25 \mathrm{~mm}$ from the wall. The heated spot expands rapidly and forms the bubble that is subsequently investigated by photographic means.

Images of the bubble are taken by a CCD camera with short exposure time (500 ns) at different times after the instant of bubble generation (Fig. 2). Image sequences of the bubble dynamics are compiled from single exposures taken of different bubbles at increasing delay time. Due to laser energy fluctuations and variations in the breakdown process the maximum radius and collapse time of the bubbles may vary slightly about their average values. Therefore, the acoustic pulses emitted at breakdown and at first collapse are measured by a hydrophone. The time between the two pulses allows to determine the collapse time precisely. Recordings of bubbles with a collapse time outside a specified range are discarded. As an example, Fig. 2 shows the collapse and rebound of a bubble having a collapse time of $140 \mu \mathrm{s}$. The first frame was acquired $10 \mu$ s after laser nucleation, the time between the frames is also 10 $\mu \mathrm{s}$.

The images are used to determine the radius-time curve $R(t)$ of the bubble (Fig. 3). As the laser-generated bubbles are, in general, not perfectly spherical an 'equivalent' radius of a sphere is calculated that gives the same cavity volume as measured on the pictures. For the example presented, a maximum radius $R_{\text {max }}=747 \mu \mathrm{m} \pm 0.5 \%$ and an upper bound for the minimum radius, $R_{\text {min }} \leq 12 \mu \mathrm{m}$, are found. At this size the measurement precision is severely limited by the optical resolution of the lenses and by image blurring due to the finite exposure time of the camera. 


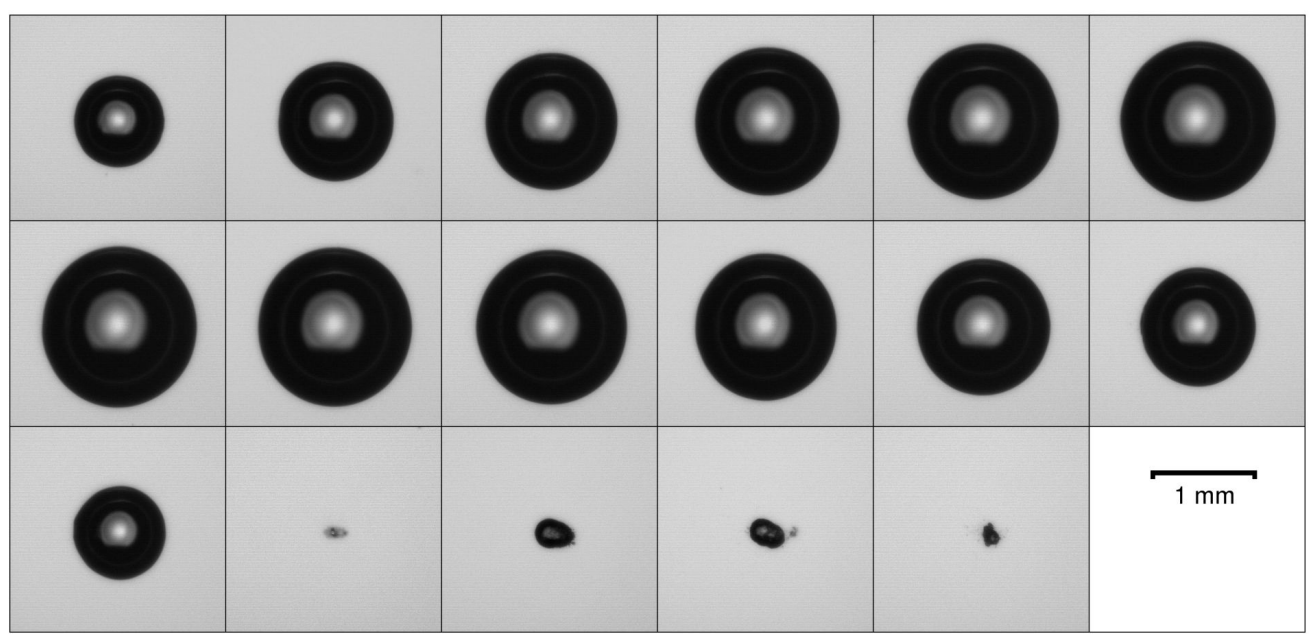

Fig. 2. Expansion and collapse of a laser-generated bubble. The exposure time is $500 \mathrm{~ns}$, the time between frames, $10 \mu \mathrm{s}$. The first image was taken $10 \mu \mathrm{s}$ after the laser shot.

\subsection{The Keller-Miksis Model}

In case of the undisturbed bubble collapse, the movement of the bubble wall characterized by the radius $R_{b}=R_{b}(t)$ can be modeled by the Keller-Miksis model for gas and vapor filled bubbles $[12,18]$. This model is derived from the Navier-Stokes equations written in spherical coordinates and employing spherical symmetry of the bubble. It is of first order in the Mach number and thus accounts for weak compressibility:

$$
\left(1-\frac{\dot{R}_{b}}{c_{0}}\right) R_{b} \ddot{R}_{b}+\frac{3}{2} \dot{R}_{b}^{2}\left(1-\frac{\dot{R}_{b}}{3 c_{0}}\right)=\left(1+\frac{\dot{R}_{b}}{c_{0}}\right) \frac{P_{R}-p_{0}}{\rho_{0}}+\frac{R_{b}}{\rho_{0} c_{0}} \dot{P}_{R}
$$

Here, $p_{0}$ denotes the ambient pressure, $\rho_{0}$, the density, and $c_{0}$ the speed of sound, which is assumed to be constant. The pressure in the liquid at the bubble wall, $P_{R}$, is given by

$$
P_{R}=p_{0}\left(\frac{R_{e q}^{3}-b R_{e q}^{3}}{R_{b}^{3}-b R_{e q}^{3}}\right)^{\kappa}
$$

where $R_{e q}$ is the equilibrium radius of the bubble, i.e., the radius at which the static bubble is at (unstable) mechanical and thermal equilibrium with the surrounding liquid. Here the terms corresponding to vapor pressure, liquid viscosity and surface tension have been neglected because there effect is negligibly small for the configurations at hand. The medium inside the bubble is described by a van der Waals hard core law with excluded volume fraction $b$ and polytropic exponent $\kappa$. 
The following numerical values were chosen for the calculations: $b=0.0016$, $\kappa=4 / 3, p_{v}=2330 \mathrm{~Pa}, c_{0}=1482 \mathrm{~m} / \mathrm{s}, \rho_{0}=998 \mathrm{~kg} / \mathrm{m}^{3}, \mu=0.001 \mathrm{~N} / \mathrm{m}^{2}$, $p_{0}=100 \mathrm{kPa}$, and $\sigma=0.0725 \mathrm{~Pa} \mathrm{~m}$.

To perform the ODE simulation we have to fix the equilibrium radius $R_{e q}$ and the initial values $t_{0}, R_{b}\left(t_{0}\right)$ and $\dot{R}_{b}\left(t_{0}\right)$. The simulation is started at $t_{0}=t_{\text {max }}$, when the bubble reaches its maximum expansion and the interface stops, thus $R_{b}\left(t_{\max }\right)=R_{\max }$ and $\dot{R}_{b}\left(t_{\max }\right)=0$. Since the measured radius-time curve is sampled at intervals of $5 \mu$ s and does not hit the maximum point exactly, values for $t_{\max }$ and $R_{e q}$ are determined by a least-squares fit, so that the calculated dynamics matches the experimental data as closely as possible. Note that the time from maximum expansion to the first collapse is nearly independent of the equilibrium radius. For $t_{\max }=70.7 \mu \mathrm{s}$ the collapse time equals the experimental value of $140 \mu \mathrm{s}$, and the equilibrium radius is found to be $R_{e q}=$ $69.2 \mu \mathrm{m}$. It can be assumed that these parameters, if not exact, give a reliable numerical approximation of the bubble's radial dynamics. In Figure 3 the numerical simulation of the Keller-Miksis model based on these parameters is compared with the experimental radius-time curve, showing good agreement. Even the first rebound is captured quite well, though the bubble tends to get distorted and shape-unstable at this stage (compare Fig. 2).

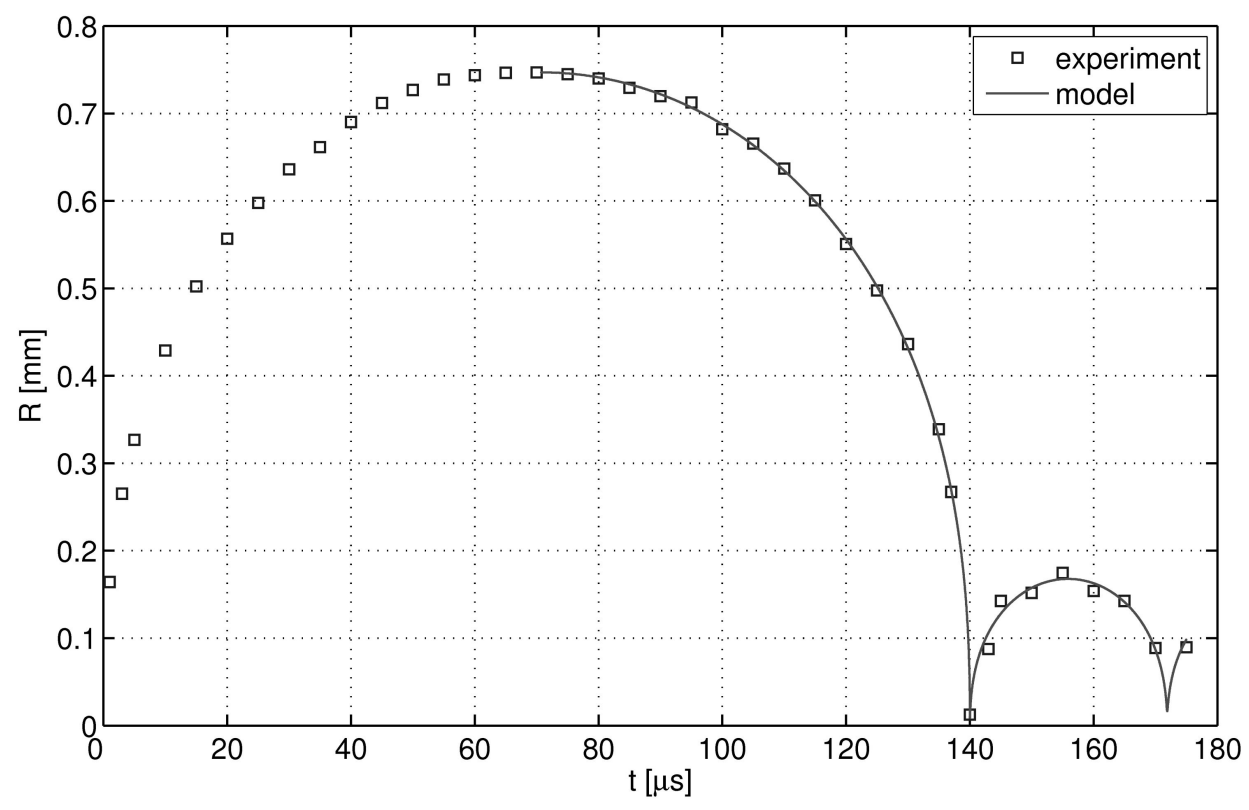

Fig. 3. The points $(\square)$ give the experimentally measured bubble radii as a function of time. The solid curve starting at maximum radius presents a numerical solution of the Keller-Miksis model with its parameters fitted to the experiment as described in the text. 


\subsection{Choice of initial gas state}

Finally we have to specify the initial state inside the bubble for a given radius $R_{b}$. Since neither the fluid equations (1) nor the Keller-Miksis model $(23,24)$ incorporate mass transfer of the non-condensable gas, the equilibrium radius $R_{e q}$ fixes the amount of gas in the bubble. $R_{e q}$ has to be be extracted from the experimental data.

In ultrasonic cavitation the gas content of oscillating bubbles typically changes slowly over many oscillation cycles due to diffusion across the interface, and accordingly depends on the concentration of dissolved gas. On the other hand, the initial gas content of laser-generated bubbles virtually does not depend on the gas concentration in the liquid, as the volume of vaporized liquid is too small to contain a significant amount of gas molecules. However, in the laser breakdown the liquid (here, water) is superheated and hydrolyzed, yielding a reactive mixture of ions (e.g., $\mathrm{H}, \mathrm{OH}, \mathrm{O}$ ) during the first nanoseconds. Upon expansion and rapid cooling of the plasma these species recombine to give mainly vapor, oxygen and hydrogen gas, and smaller amounts of other components. Oxyhydrogen gas has been shown to be produced in repetitive laser-induced breakdown in water. Unfortunately, it is not well known, and difficult to measure, how much gas is produced in the laser breakdown process compared to water vapor. However, it is safe to say that when a large laser-generated bubble reaches its maximum radius the vapor has reached phase equilibrium with the surrounding liquid, and that the amount of noncondensable gas in the bubble will remain approximately constant during the first few oscillation cycles. With the definition of the equilibrium radius,

$$
p_{i}\left(R_{e q}\right)=p_{e q}=p_{0}+\frac{2 \sigma}{R_{e q}},
$$

and assuming that the bubble medium is an ideal gas undergoing adiabatic compression or expansion, the pressure in the bubble at radius $R_{b}$ is given by

$$
p_{i}\left(R_{b}\right)=p_{e q}\left(\frac{R_{e q}}{R_{b}}\right)^{3 \kappa} \approx p_{0}\left(\frac{R_{e q}}{R_{b}}\right)^{3 \kappa}
$$

where in the last step the surface tension has been neglected, which is admissible for large enough $R_{e q}$. As the temperature in the bubble at equilibrium is the ambient temperature $T_{0}$, the inner temperature at radius $R_{b}$ is accordingly given by

$$
T_{i}\left(R_{b}\right)=T_{0}\left(\frac{R_{e q}}{R_{b}}\right)^{\kappa-1} .
$$


The mass in the bubble, $M_{i}$, is assumed to be constant, so the density in the bubble scales inversely with the volume,

$$
\rho_{i}\left(R_{b}\right)=\rho_{e q}\left(\frac{R_{e q}}{R_{b}}\right)^{3}
$$

where the equilibrium density is calculated by means of the ideal gas law,

$$
\rho_{e q}=\frac{M_{i}}{V_{e q}}=\frac{\nu W}{V_{e q}}=\frac{p_{e q} W}{\Re T_{0}} .
$$

Here, $\nu$ denotes the number of moles in the bubble, $W$ the molar mass of the gas, and $\Re=8.314 \mathrm{~J} / \mathrm{mol}$ the universal gas constant.

As an example we consider a bubble with an equilibrium radius of $R_{e q}=$ $0.692 \cdot 10^{-4} \mathrm{~m}$, filled with air $(\kappa=1.4, W=0.029 \mathrm{~kg} / \mathrm{mol})$. With an ambient temperature of $T_{0}=293.15 \mathrm{~K}$, an ambient pressure of $p_{0}=10^{5} \mathrm{~Pa}$, and liquid density of $\rho_{0}=1000 \mathrm{~kg} / \mathrm{m}^{3}$ we obtain for a bubble at radius $R_{b}=746.9 \mu \mathrm{m}$ an internal pressure of $p_{i}\left(R_{b}\right)=4.579 \mathrm{~Pa}$ and a temperature of $T_{i}\left(R_{b}\right)=16.9$ $\mathrm{K}$. The density in the bubble is $\rho_{i}\left(R_{b}\right)=0.957 \cdot 10^{-3} \mathrm{~kg} / \mathrm{m}^{3}$.

The calculated values, in particular the temperature and the pressure, appear to be unrealistic. In fact, because the vapor pressure has been neglected, they cannot faithfully represent the real conditions in the bubble at maximum expansion, as the bubble will be filled with vapor at low pressure. This vapor largely condenses when the bubble shrinks, and the remaining gas determines the evolution of pressure and density during the final collapse phase and rebound, which are at the focus of the present investigation.

\section{Results}

We investigate the collapse of a spherical bubble using the real ghost fluid method and the Saurel-Abgrall approach. This configuration can be considered as a benchmark problem to validate modeling, discretization as well as implementation, because the results can be compared with experiments and simplified 1D models. Since the flow field is inherently one-dimensional, we would like to emphasize that in $1 \mathrm{D}$ there are other models and discretizations available that might give better results for the problem at hand such as interface fitting strategies, cf. $[8,11]$ but these are not feasible for multidimensional flows or they can not deal with strong variations in the material parameters and high interface velocities

By means of this configuration we want to compare the two approaches and 
validate them by the Keller-Miksis model, which is fitted to the experimental data. In particular, we will discuss the influence of grid refinement on numerical phase transition and grid convergence. For this purpose we perform computations for two cases that only differ in the initial data for the gas.

\subsection{Case 1}

The computations have been performed on the interval $\Omega=[0,0.4] \mathrm{m}$ in radial direction. The maximum radius of the bubble is $R_{\max }=0.7469 \times 10^{-3} \mathrm{~m}$. For the initial state in the air bubble we choose $\rho_{\text {air }}=0.957 \times 10^{-3} \mathrm{~kg} / \mathrm{m}^{3}$ and $p_{\text {air }}=4.579 \mathrm{~Pa}$ according to Section 4.3. The ambient state in the liquid is determined by $\rho_{\text {water }}=1000 \mathrm{~kg} / \mathrm{m}^{3}$ and $p_{\text {water }}=10^{5} \mathrm{~Pa}$. Both phases are assumed to be at rest. The material parameters for the stiffened gas law are those given in Table 1 .

At the left boundary, i.e., the bubble midpoint, we impose symmetry conditions whereas in the far field we use reflecting boundary conditions at a wall. The reason for choosing a large computational domain is to avoid unphysical reflections at the far field boundary. The time for the numerical investigation of the phase boundary is therefore limited by twice the time needed for a wave to run from the interface to the wall and back again.

The coarse discretization consists of $N_{0}=20$ cells. The number of refinement levels for the multiscale-based grid adaptation is determined by $L$, i.e., the uniform grid on the finest level consists of $N_{L}=2^{L} N_{0}$ cells. The threshold value in the grid adaptation is chosen as $\varepsilon=10^{-4}$. This turned out to be reasonably small such that the overall accuracy of the reference scheme on the uniform fine grid was not affected by the threshold error. For details on the multiscale-based grid adaptation we refer to Refs. [3,15]. Since time discretization is explicit the time step is limited by a fixed CFL number of 0.8 in case of the SA approach and 0.2 for the rGFM for all of the presented computations.

Although the physical model, i.e., the inviscid fluid equations coupled with the stiffened gas law, is the same for both the S-A approach and the rGFM, the numerical discretization differs essentially in the approximation of the evolution equation for the phase boundary. This causes a sharp representation of the phase interface by the zero level set in the rGFM whereas in the SaurelAbgrall approach the interface is smeared due to numerical diffusion resulting in severe numerical phase transition.

Therefore we first investigate the numerical diffusion of the phase boundary using the SA approach. Since the phase boundary can not be identified by a unique value of the gas fraction $\varphi$, we choose two values $\varphi=10^{-5}$ ("pure" water) and $\varphi=1-10^{-10}$ ("pure" air) by which we characterize the limits of 


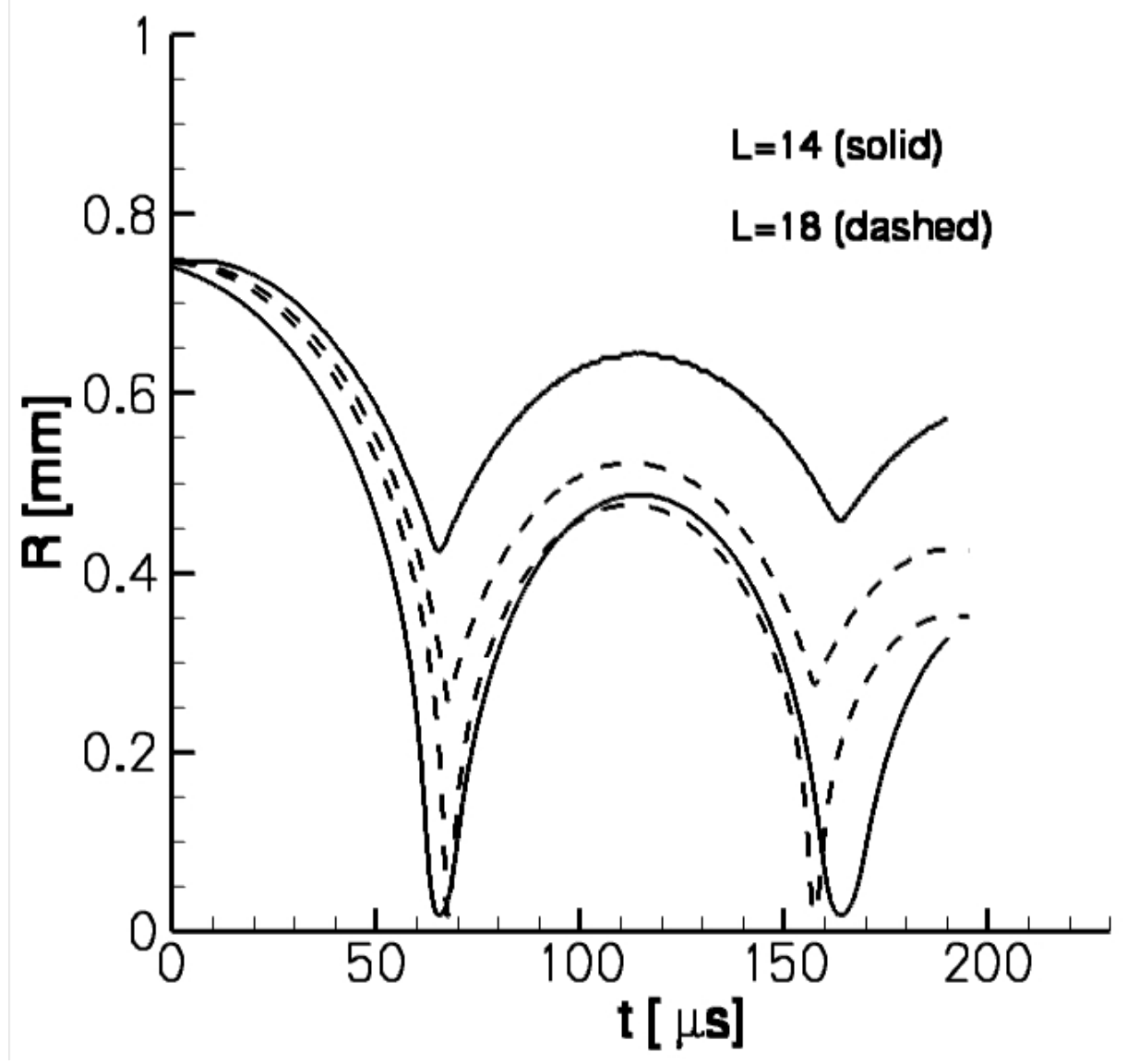

Fig. 4. Case 1: Numerical phase transition of SA approach

the pure phases. In Figure 4 we present the two isolines for the gas fraction corresponding to different computations with increasing refinement level $L$. We note that these isolines are diverging until the first rebound. The distance reaches its maximum when the bubble radius becomes minimal. Thereafter the isolines are converging again as long as the bubble is growing in the rebound. The thickness of the numerical phase transition regime is severe. However, it decreases significantly under grid refinement. Hence, we conclude that this regime might vanish under further grid refinement. However, as is seen from Table 2 the thickness $\Delta R$ of the phase transition zone measured at the first rebound is converging numerically of order 1 with a reduction factor of about $c=0.8$. To reduce the zone thickness of $\Delta R_{L}=59 \mu \mathrm{m}$ at $L=18$ to a size comparable to the minimum size of the bubble at first collapse as given by the Keller-Miksis model (Table 3), $R_{\min }=10 \mu \mathrm{m}$, we have to reduce the thickness by another factor of $\Delta R_{L} / R_{\text {min }}=\Delta R_{L} / \Delta R_{L+1} \approx 6$, i.e. we need $l \geq \log \left(\Delta R_{L+l} / \Delta R_{L}\right) / \log (c) \approx 8$ additional refinement levels. Since by each additional refinement level the computational time increases by a factor of about 3 , a computation on level $L=26$ would be about 6600 times longer. 
Table 2

Case 1: Grid convergence of numerical phase transition regime at the 1st rebound

\begin{tabular}{|l|l|l|l|l|l|l|}
\hline$L$ & 13 & 14 & 15 & 16 & 17 & 18 \\
\hline$\Delta R[\mathrm{~mm}]$ & 0.176 & 0.169 & 0.129 & 0.099 & 0.072 & 0.059 \\
\hline$p=\log \Delta R_{L} / \log \Delta R_{L-1}$ & - & 1.023 & 1.152 & 1.129 & 1.138 & 1.076 \\
\hline$c=\Delta R_{L} / \Delta R_{L-1}$ & - & 0.960 & 0.763 & 0.767 & 0.727 & 0.819 \\
\hline
\end{tabular}

This is not feasible.

Next we want to discuss the influence of grid refinement on the phase boundary where we identify the bubble wall by the gas fraction $\varphi=1-10^{-10}$ ("pure" air) in the SA approach and use the zero level set $\Phi=0$ for the rGFM. In Figures 5 and 6 the moving bubble wall is shown for several computations using an increasing number of refinement levels. We note that in the first collapse and the first rebound the bubble radius seems to be grid converged. However, in the second rebound we still can observe a significant difference with increasing refinement levels. This observation holds true for both methods.

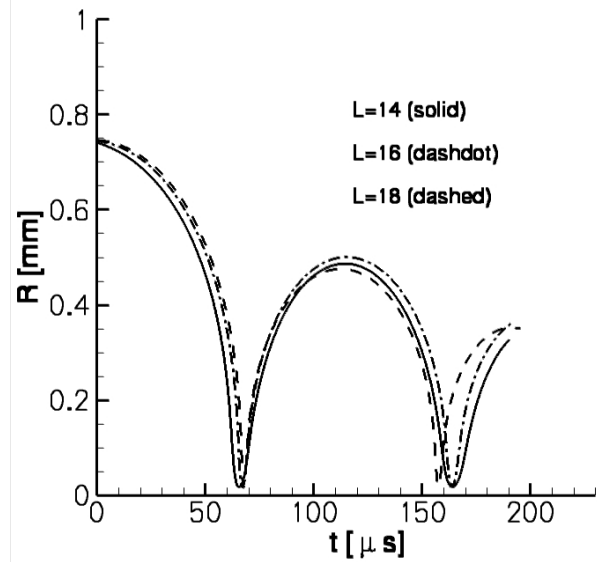

Fig. 5. Case 1: Parameter study with SA approach w.r.t. moving bubble wall

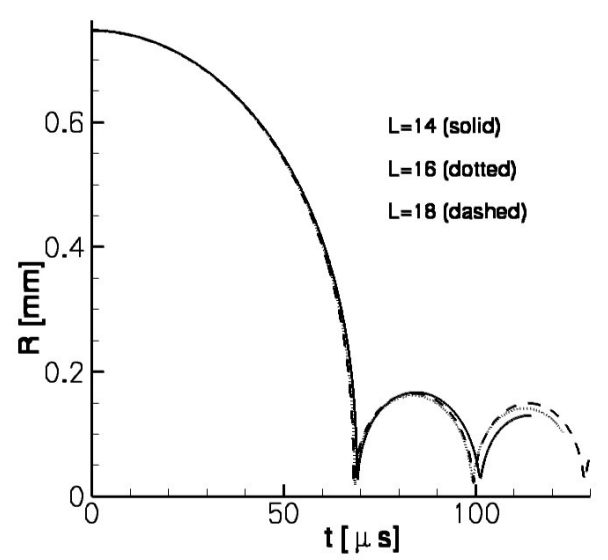

Fig. 6. Case 1: Parameter study with rGFM w.r.t. moving bubble wall

Next the numerical results are validated by means of the Keller-Miksis model introduced in Section 4.2 and fitted to the experimental data as described in Section 4.3. For this purpose, we present in Figure 7 the computations with $L=18$ refinement levels for the SA approach and the rGFM as well as the curve obtained with the Keller-Miksis model, shown in Fig. 3. We observe a good agreement for the rGFM. For the SA approach the rebound is significantly overpredicted. Obviously, the numerical phase transition regime still has a strong influence on the computation and further grid refinement is needed to reduce its influence on the rebound. However, this will exhaust available computational resources as has been discussed above, in particular, 
for genuinely multidimensional computations.

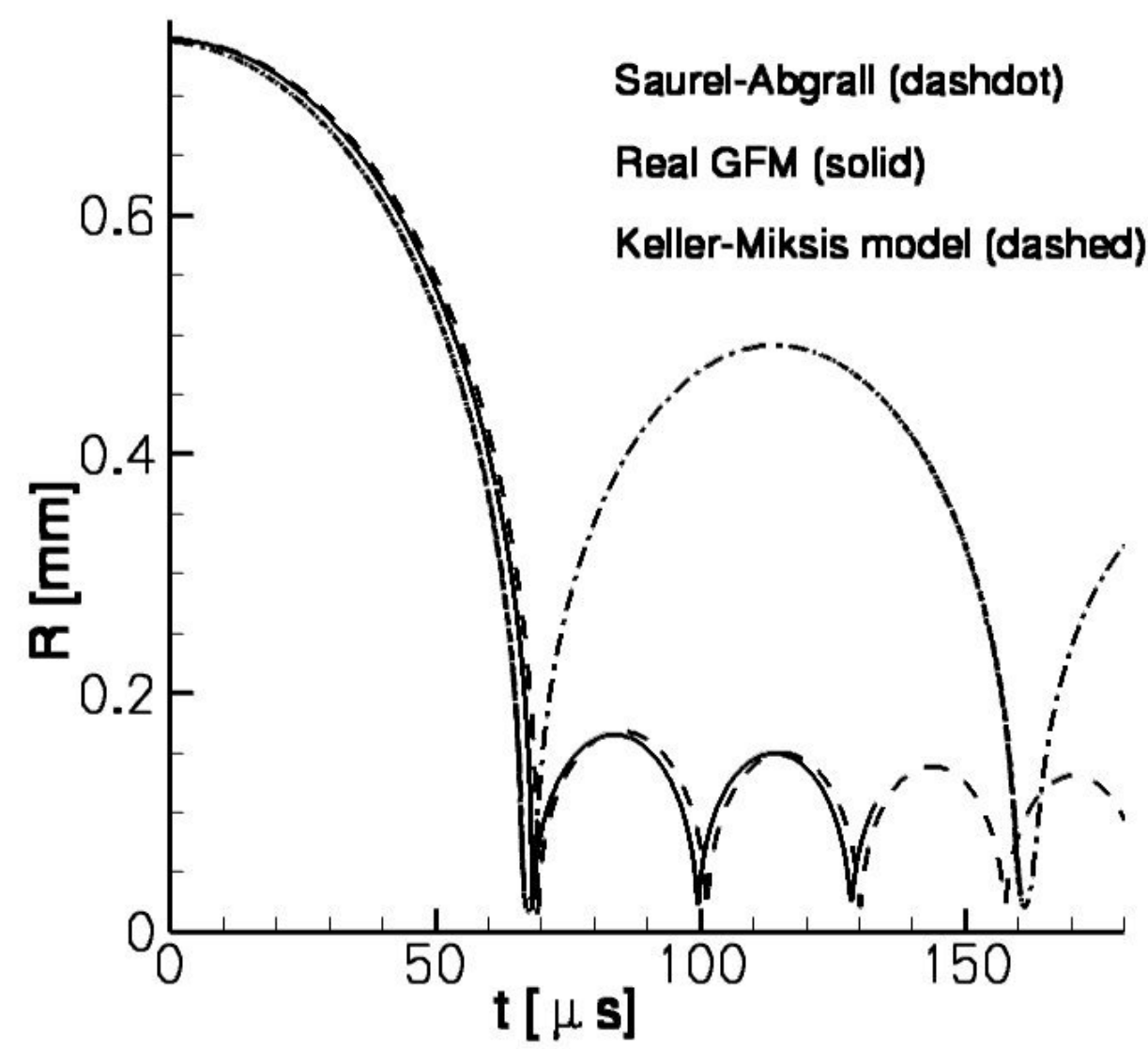

Fig. 7. Case 1: Validation of numerical methods with $L=18$ for Eqs. $(1,2)$ by comparison with the solution of the Keller-Miksis model (Eqs. $(23,24)$ ) that gives best fit to the experimental data.

For a quantitative comparison we summarize in Table 3 the bubble radius and the pressure in the gas for the instant of the first and second collapse as well as the first rebound. Several computations with increasing number of refinement levels with the SA approach and the rGFM have been performed and are compared with the Keller-Miksis model. Since the Keller-Miksis model has been tuned to the experiment, see Section 4, we suppose that this model gives the most realistic values, at least the order of magnitude should be reliable. We note a fairly good agreement in the first bubble collapse time. However, in comparison to the Keller-Miksis model, the pressure is underpredicted by the SA approach and the rGFM due to the fact that the minimum bubble radius is overpredicted by a factor of about 2. For the first rebound the rGFM and the Keller-Miksis model are in good agreement, whereas the maximum bubble radius is overpredicted by the SA approach resulting in a very low pressure. Of course, this affects significantly the second collapse that makes a compar- 
Table 3

Case 1: Comparison of Saurel-Abgrall approach, the real ghost fluid method and the Keller-Miksis model at the instance of the 1st and 2nd collapse as well as the 1st rebound corresponding to the minimum and maximum bubble size

\begin{tabular}{|c|l||c|c|c||c|c|c||c|}
\hline \multicolumn{2}{|c||}{} & \multicolumn{2}{c||}{ Saurel-Abgrall Approach } & \multicolumn{2}{c||}{ Real Ghost Fluid Method } & K-M \\
\multicolumn{2}{|c||}{ Levels of refinement } & L14 & L16 & L18 & L14 & L16 & L18 & Model \\
\hline \hline \multirow{2}{*}{ 1st } & time $\mu \mathrm{s}$ & 65.4 & 67.0 & 67.7 & 69.2 & 68.5 & 68.5 & 69.3 \\
collapse & radius $\mu \mathrm{m}$ & 17.5 & 17.54 & 15.9 & 25.8 & 17.5 & 18.7 & 9.2 \\
& pressure $\mathbf{1 0}^{5} \mathbf{P a}$ & 87.0 & 196 & 410 & 101 & 398 & 300 & 1510 \\
\hline
\end{tabular}

\begin{tabular}{|c|c|c|c|c|c|c|c|c|}
\hline $\begin{array}{l}\text { 1st } \\
\text { rebound }\end{array}$ & $\begin{array}{l}\text { time } \mu \mathrm{s} \\
\text { radius } \mu \mathrm{m} \\
\text { pressure } \mathbf{P a}\end{array}$ & $\begin{array}{c}114.3 \\
487 \\
4\end{array}$ & $\begin{array}{c}115.2 \\
500 \\
12\end{array}$ & $\begin{array}{c}112.7 \\
476 \\
24\end{array}$ & $\begin{array}{c}84.5 \\
167 \\
3450\end{array}$ & $\begin{array}{c}83.6 \\
162 \\
3226\end{array}$ & $\begin{array}{c}83.7 \\
165 \\
3140\end{array}$ & $\begin{array}{c}85.8 \\
168 \\
4348\end{array}$ \\
\hline $\begin{array}{c}\text { 2nd } \\
\text { collapse }\end{array}$ & $\begin{array}{l}\text { time } \mu \mathrm{s} \\
\text { radius } \mu \mathrm{m} \\
\text { pressure } \mathbf{1 0}^{5} \mathbf{P a}\end{array}$ & $\begin{array}{c}163.8 \\
17.2 \\
41\end{array}$ & $\begin{array}{c}163.8 \\
20.1 \\
83\end{array}$ & $\begin{array}{c}157.5 \\
19.8 \\
147\end{array}$ & $\begin{array}{c}101.2 \\
29.5 \\
58\end{array}$ & $\begin{array}{c}99.3 \\
22.6 \\
130\end{array}$ & $\begin{array}{c}99.5 \\
22.0 \\
150\end{array}$ & $\begin{array}{c}101.2 \\
16.4 \\
369\end{array}$ \\
\hline
\end{tabular}

ison obsolete. For the rGFM and the Keller-Miksis model the agreement is still fairly good. Finally for the collapses, the SA approach gives a better approximation of the minimum radius than the rGFM but needs more levels of refinement to obtain an accurate pressure corresponding to this radius.

To conclude with the discussion we point out the main benefit of the Euler equations (1) in comparison to the simplified model (23) of Keller and Miksis. The spatial discretization of (1) in the SA and rGFM approach allows to represent the evolving inhomogeneous state of the medium inside the bubble during collapse and rebound, whereas the simplified model (23) is based on the approximation of a spatially homogeneous state. In Figures 8 and 9 we show the Mach number distribution for the computations with $L=14$ refinement levels using the SA approach and the rGFM. Both computations show a compression wave running inside the bubble that is reflected in the bubble center and interacts with the bubble wall. The waves travel back and forth several times in the first collapse resulting in an acceleration of the bubble wall. The main difference in the two computations is the interface velocity that becomes supersonic for the rGFM but remains subsonic for the SA approach.

\subsection{Case 2}

In this case the computational setup is identical to the previous case except for the initial data of the gas. These are determined according to Section 4.3 where we assume an equilibrium radius that is 10 times larger than determined in Section 4.2, i.e., $R_{e q}=0.692 \times 10^{-3} \mathrm{~m}$. From this radius we deduce $\rho_{\text {air }}=0.957$ $\mathrm{kg} / \mathrm{m}^{3}$ and $p_{\text {air }}=72.56 \mathrm{kPa}$. Since the bubble size will oscillate around this radius, the minimal bubble radius reached in the bubble collapse will be much larger than before and the influence of numerical phase transition will not be as severe. In addition, grid convergence should be observed already for a 


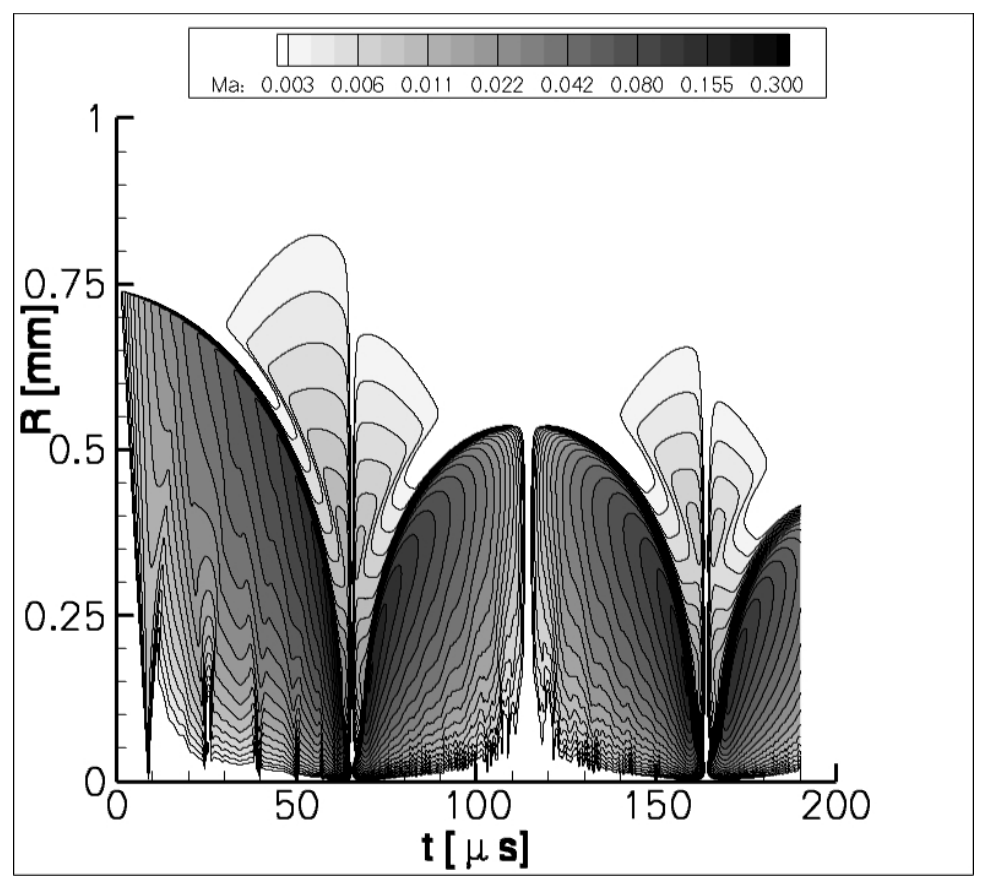

Fig. 8. Case 1: Mach number with SA approach on $L=14$

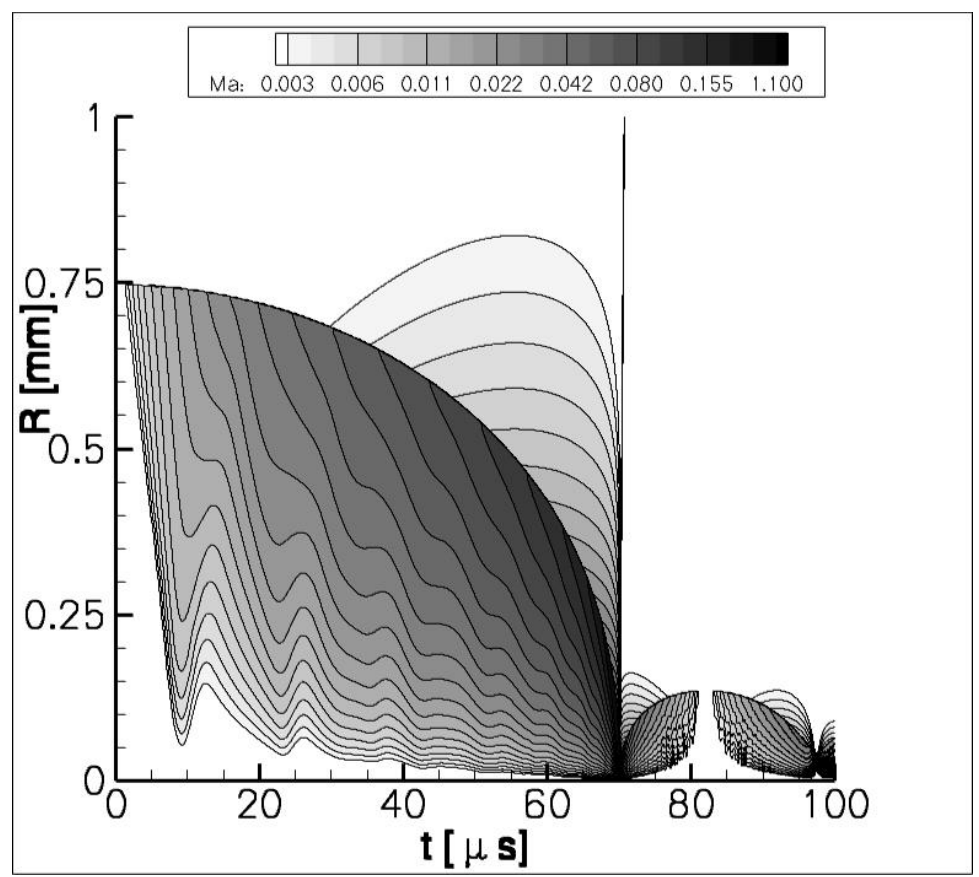

Fig. 9. Case 1: Mach number with rGFM on $L=14$

moderate number of refinement levels. By this test case it will be possible to verify numerically that the SA approach and the rGFM will converge to the same solution under grid refinement.

First of all, we investigate the numerical diffusion of the phase boundary applying the SA approach to this test case. In Figure 10 we present the two 


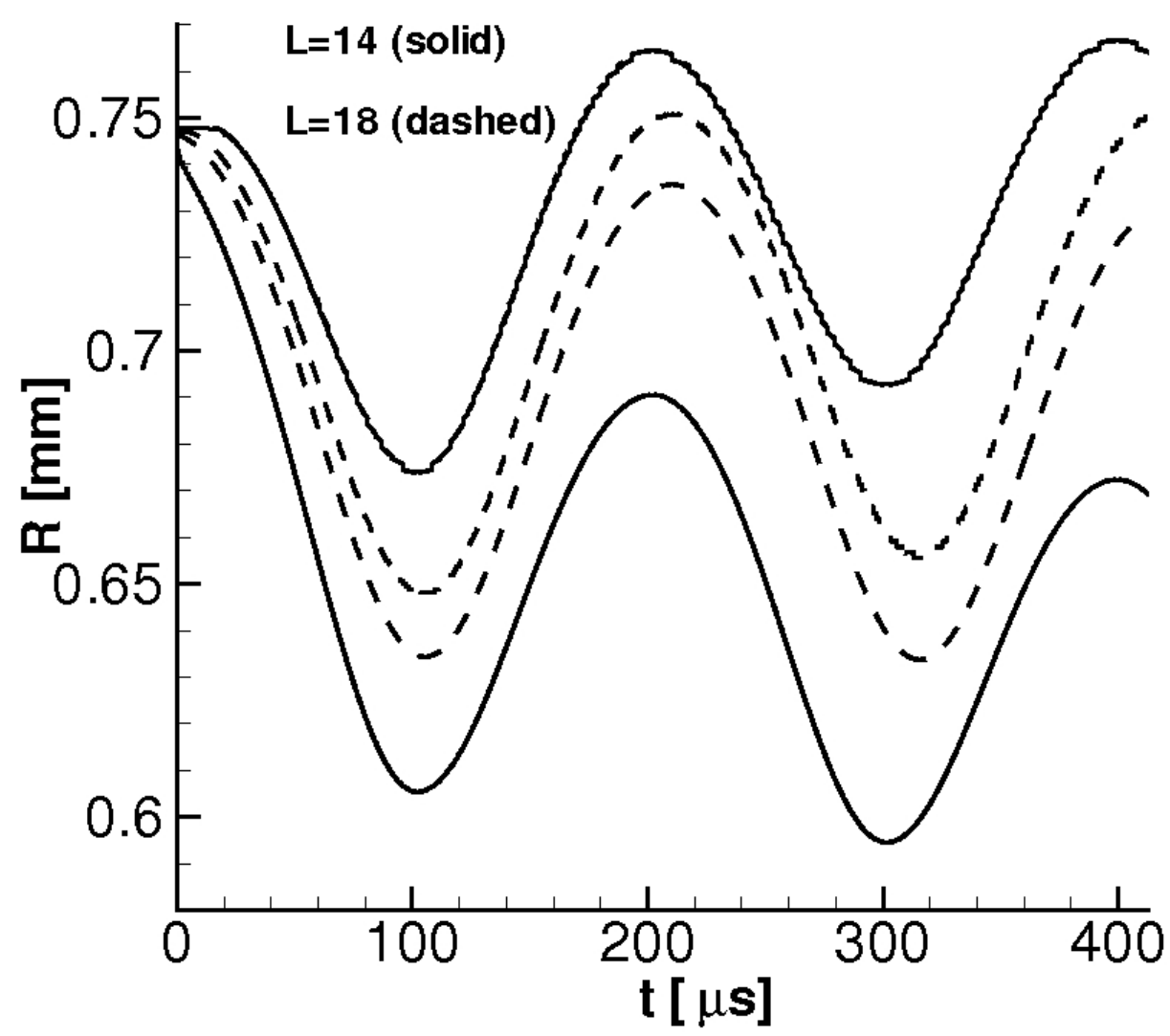

Fig. 10. Case 2: Numerical phase transition of SA approach

isolines for the gas fraction as in test case 1 corresponding to different computations for two refinement levels $L$. We note that the isolines for the two pure phases are still diverging but less severe as in case 1, see Figure 4 and Table 4. Obviously, the numerical phase transition regime is decreasing significantly under grid refinement and is approaching a sharp interface. In order to quantify this observation we again compute the empirical order of accuracy where we measure the thickness $\Delta R$ of the phase transition zone at the first rebound. As we can conclude from Table $4, \Delta R$ is converging numerically of order 1 with a reduction factor of about $c=0.7$. Note that the thickness $\Delta R_{18}=15.1 \mu \mathrm{m}$ of the transition zone is of the order of the minimum bubble size in the first collapse, see Table 5, i.e., additional refinement levels are not needed to meet the tolerance as in the previous case. Therefore the effect of the numerical phase transition regime on the bubble collapse is not as strong as in test case 1.

The influence of grid refinement on the phase boundary is shown for several computations using an increasing number of refinement levels for both approaches, see Figures 11 and 12. In the first collapse and the first rebound the 
Table 4

Case 2: Grid convergence of numerical phase transition regime at the 1st rebound

\begin{tabular}{|l|l|l|l|l|l|l|}
\hline$L$ & 13 & 14 & 15 & 16 & 17 & 18 \\
\hline$\Delta R[\mathrm{~mm}]$ & 0.1116 & 0.0737 & 0.0483 & 0.0320 & 0.0219 & 0.0151 \\
\hline$p=\log \Delta R_{L} / \log \Delta R_{L-1}$ & - & 1.189 & 1.162 & 1.136 & 1.110 & 1.097 \\
\hline$c=\Delta R_{L} / \Delta R_{L-1}$ & - & 0.660 & 0.655 & 0.663 & 0.684 & 0.689 \\
\hline
\end{tabular}

solution seems to be almost grid converged on $L=18$. This is more obvious for the rGFM but for the SA approach the difference to $L=17$ (not shown here) is small. However, in the second rebound we still can observe a significant difference with an increasing number of refinement levels. This observation holds true for both methods.

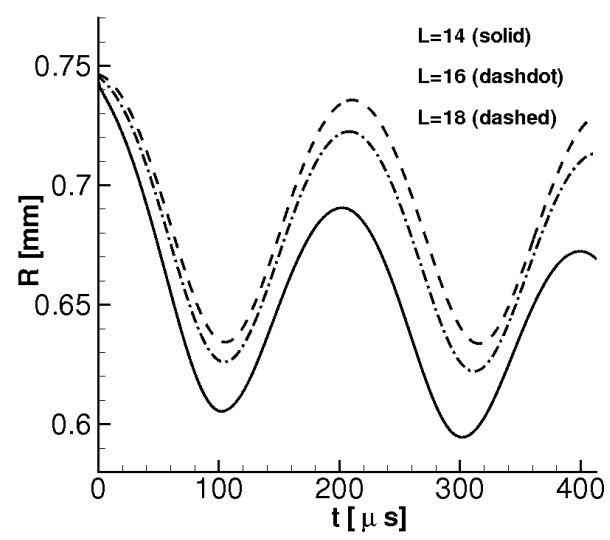

Fig. 11. Case 2: Parameter study with SA Fig. 12. Case 2: Parameter study with approach w.r.t. moving bubble wall

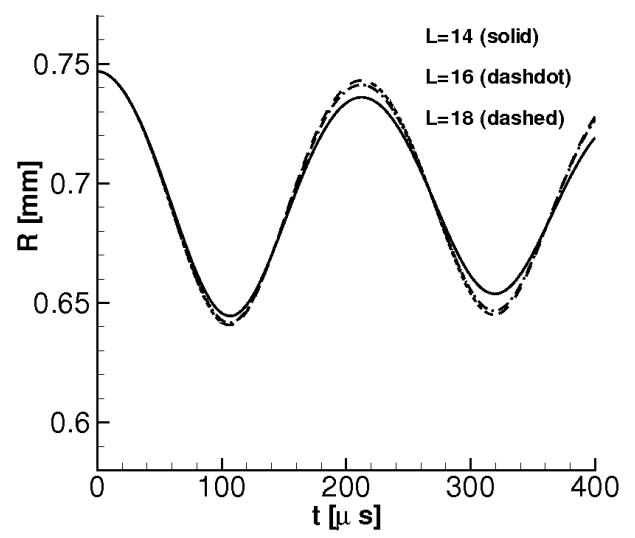

rGFM w.r.t. moving bubble wall

Opposite to test case 1, shown in Fig. 7, both schemes seem to converge to the same limit. In particular, the phase interface computed by the rGFM fully lies in the numerical phase transition regime determined by the SA approach. This holds true for all of our computations corresponding to $L=13, \ldots, 18$ for test case 2. Exemplarily, it is shown for $L=18$ in Fig. 13.

The results by the rGFM and the SA approach are both in fairly good agreement with the solution of the Keller-Miksis model, see Figure 14. However, there is a slight phase shift in time in the first collapse and rebound that is increasing with time. This is due to the different modeling. Since the KellerMiksis model (23) is a simplified model derived from the fluid equations of continuum mechanics, it can not be considered the limit of the rGFM and the SA approach discretizing the fluid equations (1) where viscosity and heat conduction are neglected. In particular, the Keller-Miksis model does not take into account the inhomogeneity of the gas state. 


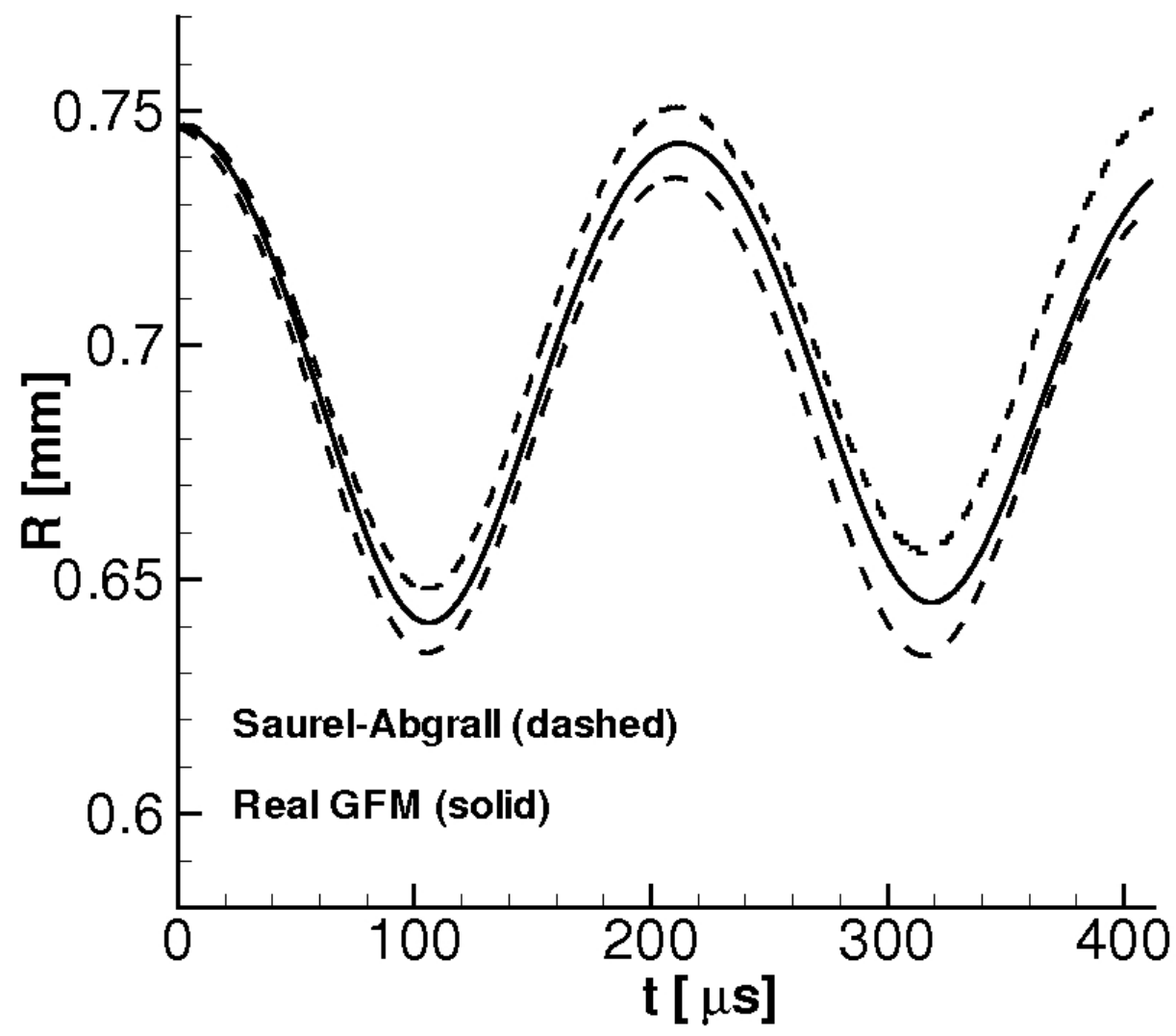

Fig. 13. Case 2: Comparison of phase boundary (rGFM) and the numerical phase transition regime (SA approach) for $L=18$

Table 5

Case 2: Comparison of Saurel-Abgrall approach, the real ghost fluid method and the Keller-Miksis model at the instance of the 1st and 2nd collapse as well as the 1st rebound corresponding to the minimum and maximum bubble size

\begin{tabular}{|c|l||c|c|c||c|c|c||c|}
\hline \multicolumn{2}{|c||}{} & \multicolumn{3}{c||}{ Saurel-Abgrall Approach } & \multicolumn{3}{c||}{ Real Ghost Fluid Method } & K-M \\
\multicolumn{2}{|c|}{ Levels of refinement } & L14 & L16 & L18 & L14 & L16 & L18 & Model \\
\hline \hline \multirow{2}{*}{ 1st } & time $\mu \mathrm{s}$ & 103 & 104 & 105 & 107 & 106 & 106 & 110 \\
collapse & radius $\mu \mathrm{m}$ & 606 & 626 & 634 & 644 & 642 & 641 & 640 \\
& pressure $\mathbf{1 0}^{\mathbf{5}} \mathbf{P a}$ & 1.38 & 1.39 & 1.39 & 1.35 & 1.37 & 1.38 & 1.36 \\
\hline
\end{tabular}

\begin{tabular}{|c|l||c|c|c||c|c|c||c|}
\hline 1st & time $\mu \mathrm{s}$ & 203 & 208 & 211 & 212 & 212 & 212 & 220 \\
rebound & radius $\mu \mathrm{m}$ & 691 & 723 & 736 & 736 & 741 & 743 & 745 \\
& pressure $\mathbf{1 0}^{\mathbf{5}} \mathbf{P a}$ & 0.74 & 0.74 & 0.74 & 0.77 & 0.75 & 0.74 & 0.75 \\
\hline
\end{tabular}

\begin{tabular}{|c|l||c|c|c||c|c|c||c|}
\hline 2nd & time $\mu \mathrm{s}$ & 302 & 312 & 316 & 319 & 319 & 319 & 330 \\
collapse & radius $\mu \mathrm{m}$ & 594 & 622 & 634 & 654 & 647 & 645 & 642 \\
& pressure $\mathbf{1 0}^{5} \mathbf{P a}$ & 1.34 & 1.36 & 1.37 & 1.27 & 1.33 & 1.34 & 1.34 \\
\hline
\end{tabular}

The agreement is confirmed by the quantitative comparison in Table 5 for the bubble radius and the pressure in the gas for the instant of the first and second collapse as well as the first rebound. 


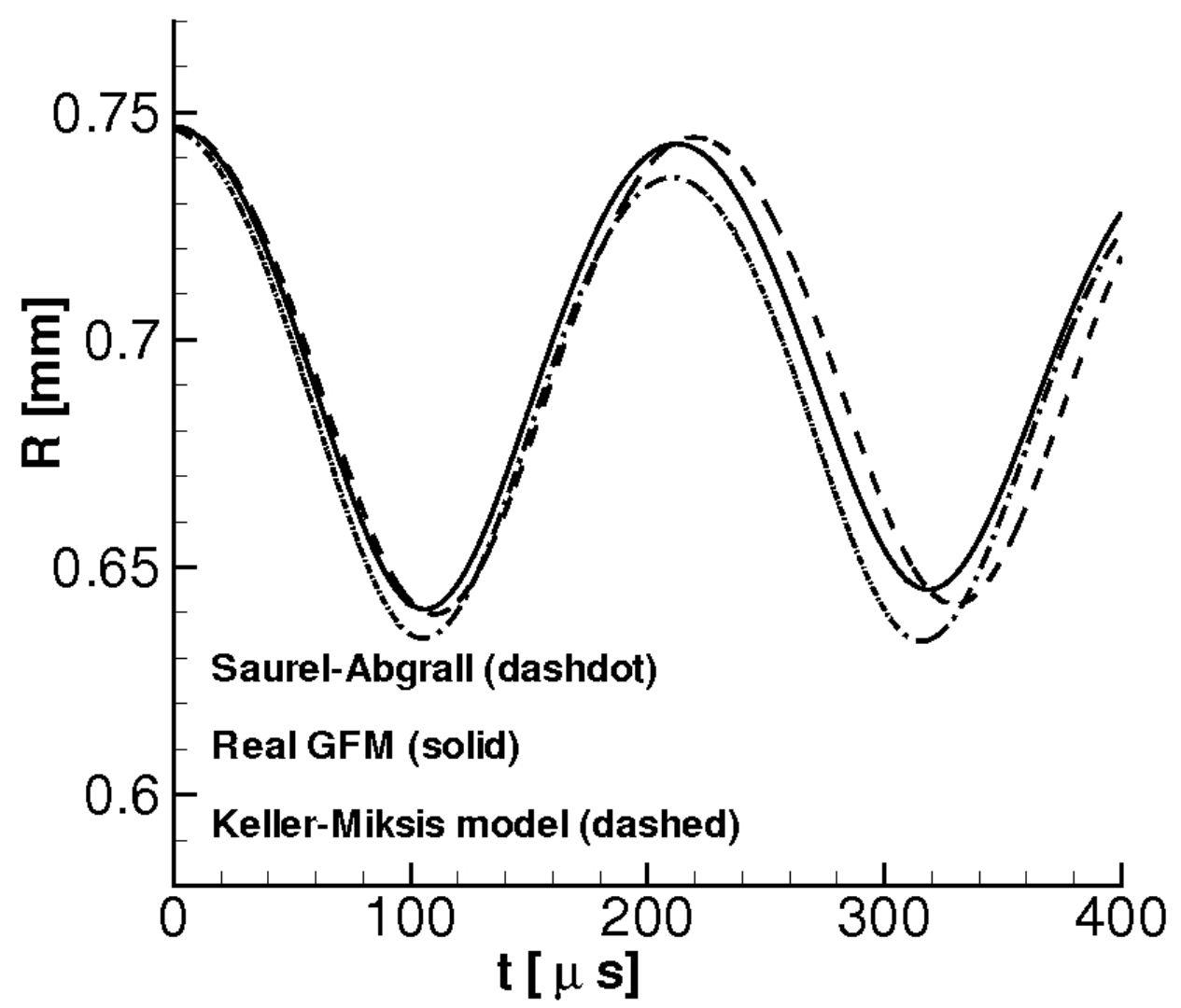

Fig. 14. Case 2: Validation of numerical methods by the Keller-Miksis model

Finally, Figures 15 and 16 show the Mach number distribution for the computations with $L=14$ refinement levels using the SA approach and the rGFM, respectively. For both computations we note that the bubble wall is only weakly accelerated and stays subsonic. In particular, the Mach number inside the bubble is much smaller than in case 1. At a Mach number less than 0.005 the fluid can be considered incompressible. Therefore the compression wave is running much faster relative to the gas velocity and, hence, the bubble wall, i.e., the process of reflection in the bubble center and interaction at the bubble wall takes place more frequently. This causes the wiggles in the isolines. Note that both computations give qualitatively comparable results.

\section{Conclusion}

The SA approach and the rGFM have been applied to investigate the spherical bubble collapse of laser-induced cavitation bubbles. Initial data are derived by first fitting the equilibrium radius in the Keller-Miksis model to minimize the 


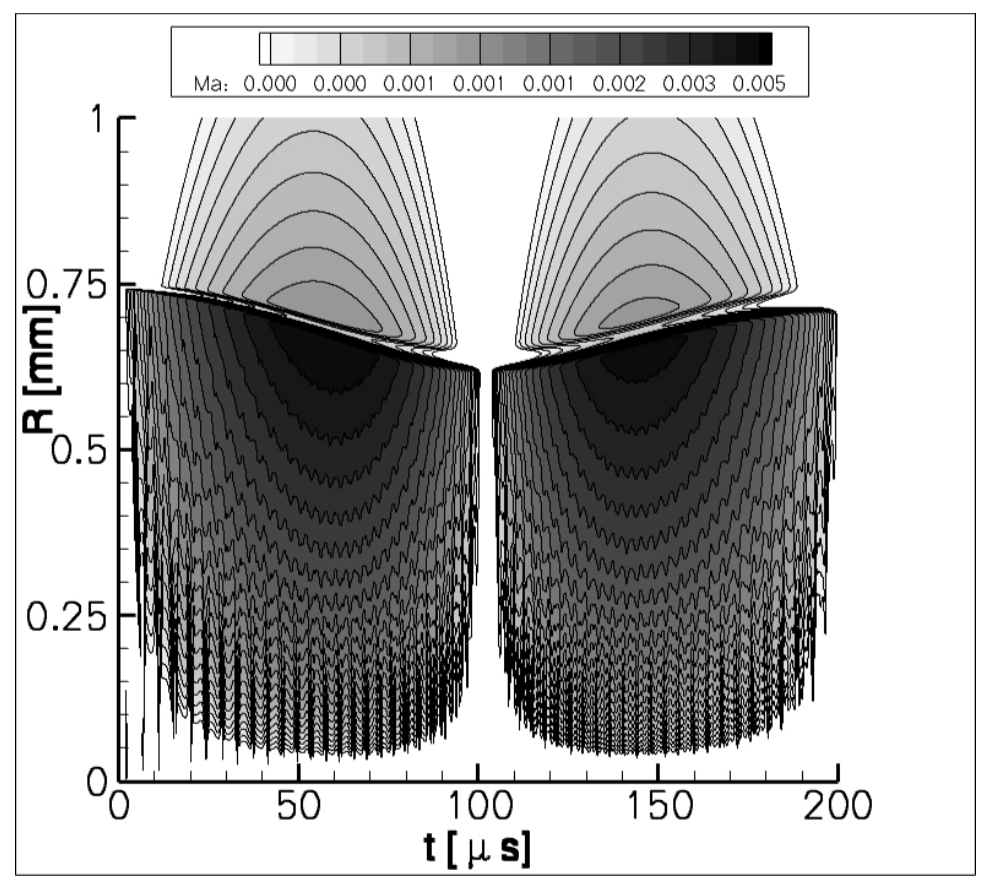

Fig. 15. Case 2: Mach number with SA approach on $L=14$

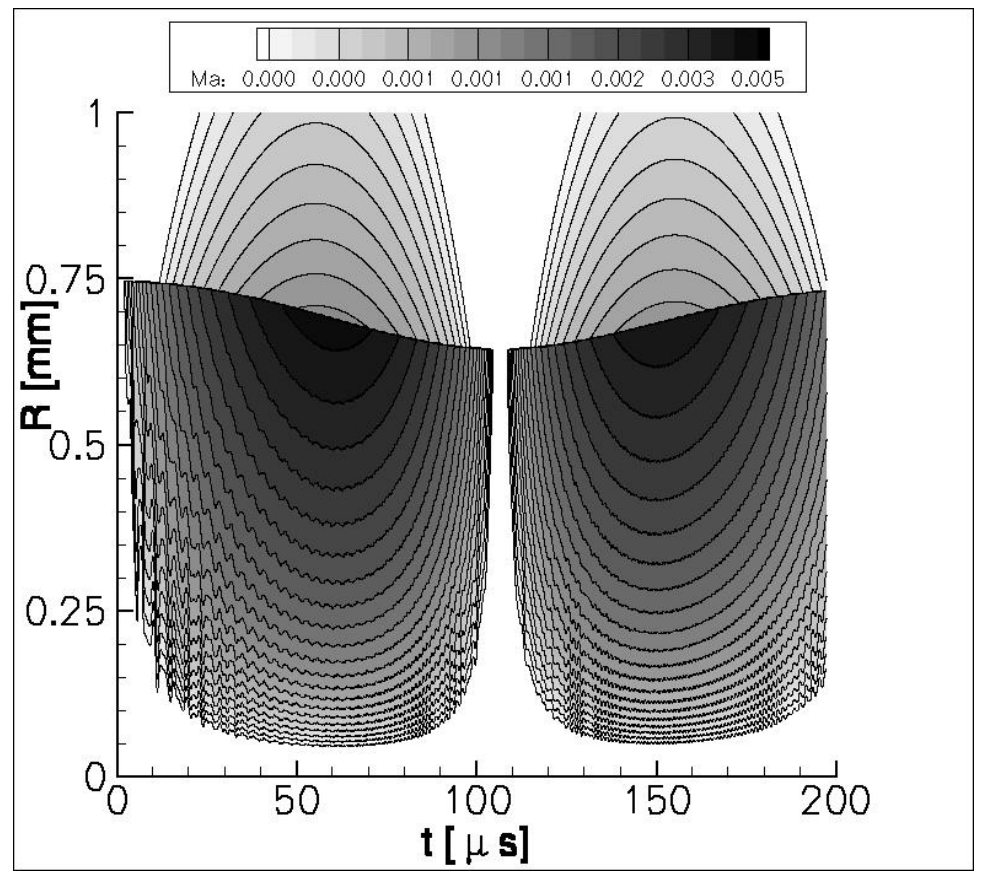

Fig. 16. Case 2: Mach number with rGFM on $L=14$

least-squares error of the model solution relative to the experimental data on the bubble dynamics. Then the gas state for an arbitrary bubble radius is deduced applying an ideal and adiabatic gas law.

The CFD computations verify that the SA approach results in a severe numerical phase transition regime that is unphysical because mass transfer is 
excluded in the physical model. This regime can be reduced by grid refinement but grid convergence is too slow and the needed resolution to reduce this effect significantly can not be afforded. On the other hand, the rGFM leads to fairly good results that are comparable to experimental data even for a moderate resolution.

Therefore we conclude that a sharp interface model is needed to obtain physically reasonable results for the investigation of laser-induced cavitation bubbles. In particular, the smearing of the phase boundary has to be avoided to reduce the influence of numerical mass transfer between the two phases. Hence, future work is concerned with the implementation of the rGFM in multidimensions.

\section{References}

[1] R. Abgrall, How to prevent pressure oscillations in multicomponent flow calculations: A quasi-conservative approach, J. Comp. Phys., 125 (1996), 150160 .

[2] I. Akhatov, O. Lindau, A. Topolnikov, R. Mettin, V. Vakhitova, W. Lauterborn, Collapse and rebound of a laser-induced cavitation bubble, Phys. Fluids, 13, No. 10 (2001), 2805-2819.

[3] S. Andreae, Wave Interactions with Material Interfaces, Dissertation, RWTH Aachen, Shaker-Verlag, 2008.

[4] T. Barberon, Ph. Helluy, Finite volume simulation of cavitating flows, Computers and Fluids, 34, No. 7 (2005), 832-858.

[5] T. Barberon, Ph. Helluy, S. Rouy, Practical computation of axisymmetrical multifluid flows, International Journal of Finite Volumes, 1, No. 1 (2003), 1-34.

[6] C.-H. Chang, M.-S. Liou, A robust and accurate approach to computing compressible multiphase flow: Stratified flow model and $\mathrm{AUSM}^{+}$-up scheme, J. Comp. Phys., 225 (2007), 840-873.

[7] J.-P. Cocchi, R. Saurel, A Riemann problem based method for the resolution of compressible multimaterial flows, J. Comp. Phys., 137, No. 2 (1997), 265-298.

[8] M. Hanke, J. Ballmann, Strong Changes of State in Collapsing Bubbles, ZAMM, 78, Suppl. 1 (1998), 453-454.

[9] F. Harlow, A. Amsden, Fluid dynamics, Los Alamos National Laboratory, Technical Report, LA-4700, 1971.

[10] A. Harten, B. Engquist, S. Osher, S.R. Chakravarthy, Uniformly high order accurate Essentially Non-Oscillatory Schemes. III., J. Comp. Phys., 71 (1987), 231-303. 
[11] P. Helluy, H. Mathis, S. Müller, An ALE averaging approach for the computing of bubble oscilations, in Finite volumes for complex applications V, R. Eymard, J.-M. Hérard (eds.), Wiley, 487-494, 2008.

[12] J.B. Keller, M. Miksis, Bubble oscillations of large amplitude, J. Acoust. Soc. Am., 68, No. 2 (1980), 628-633.

[13] W. Lauterborn, Numerical investigation of nonlinear oscillations of gas bubbles in liquids, J. Acoust. Soc. Am., 59, No. 2 (1976), 283-293.

[14] W. Lauterborn, Th. Kurz, R. Mettin, C.-D. Ohl, Experimental and theoretical bubble dynamics, Advances in Chemical Physics, 110 (1999), 295-380.

[15] S. Müller, Ph. Helluy, J. Ballmann, Numerical Simulation of Cavitation Bubbles by Compressible Two-Phase Fluids, RWTH Aachen, IGPM-Report 159, 2007.

[16] R.R. Nourgaliev, T.N. Dinh, T.G. Theofanous, Adaptive characteristics-based matching for compressible multifluid dynamics, J. Comp. Phys., 213 (2006), $500-529$.

[17] S. Osher, R. Fedkiw, Level set methods and dynamic implicit surfaces, Applied Mathematical Sciences, 153, Springer, New York, 2003.

[18] A. Prosperetti, A. Lezzi, Bubble dynamics in a compressible liquid. I. Firstorder theory, J. Fluid. Mech., 168 (1986), 457-478.

[19] R. Saurel, R. Abgrall, A simple method for compressible multifluid flows, SIAM J. Sci. Comput., 21, No. 3 (1999), 1115-1145.

[20] J.A. Sethian, Level set methods and fast marching methods. Evolving interfaces in computational geometry, fluid mechanics, computer vision, and materials science, Cambridge Monographs on Applied and Computational Mathematics, 3, Cambridge University Press, 1999.

[21] C.W. Shu, S. Osher, Efficient implementation of essentially nonoscillatory shock-capturing schemes. II., J. Comp. Phys., 83 (1989), 32-78.

[22] U. Specht, Numerical Simulation of Mechanical Waves at Fluid-Solid-Interfaces, Dissertation, RWTH Aachen, VDI-Verlag, Reihe 7, 2000 (in German).

[23] M. Sussman, P. Smereka, S. Osher, A level set approach for computing solutions to incompressible two-phase flows, J. Comp. Phys., 114 (1994), 146-159.

[24] M. Sussman, A.S. Almgren, J.B. Bell, P. Colella, L.H. Howell, M.L. Welcome, An adaptive level set approach for incompressible two-phase flows, J. Comp. Phys., 148 (1999), 81-124.

[25] C.W. Wang, T.G. Liu, B.C. Khoo, A real ghost fluid method for the simulation of multimedium compressible flow, SIAM J. Sci.. Comput., 28, No. 1 (2006), $278-302$. 